\title{
Offside Detection System Using an Infrared Camera Tracking System
}

\author{
Esteban Lopez, Peter E. Jenkins* \\ Mechanical Engineering Department, University of Colorado, Denver, USA \\ Email: *peter.jenkins@ucdenver.edu
}

How to cite this paper: Lopez, E. and Jenkins, P.E. (2019) Offside Detection System Using an Infrared Camera Tracking System. World Journal of Mechanics, 9, 163-176. https://doi.org/10.4236/wjm.2019.96011

Received: May 29, 2019

Accepted: June 25, 2019

Published: June 28, 2019

Copyright $\odot 2019$ by author(s) and Scientific Research Publishing Inc. This work is licensed under the Creative Commons Attribution International License (CC BY 4.0).

http://creativecommons.org/licenses/by/4.0/

\section{c) (i) Open Access}

\begin{abstract}
This paper describes an experimental offside detection system that will be capable of detecting offside passes during a game of soccer. Soccer is the world's most popular and most televised sport. In recent years, FIFA has implemented goal line technology in order to end controversial goals/missed goals during high profile competitive matches. The most contentious aspect of the sport is the offside rule and its many controversial calls or lack of calls. Sometimes the linesmen cannot see the passage of playing fast enough to make a correct decision. Being similar to goal line technology, people have requested offside technology to help the linesmen and to reduce the number of incorrect offside calls in a game. This paper describes a working offside detection system that can accurately detect offside passes. Positional data was exported from a VICON infrared motion tracking camera system and a MATLAB script was written so that it can analyze the positions of the players and the ball and determine if a pass was offside.
\end{abstract}

\section{Keywords}

Soccer, Motion Simulation

\section{Introduction}

The purpose of this project is to design and develop an experimental offside detection system that will be capable of detecting offside passes during a game of soccer. Soccer is the world's most popular and most televised sport. Every year, thousands of players from all across the world compete in some of the most competitive and prestigious leagues. In recent years, FIFA [1], soccer's governing body, has implemented goal line technology in order to end controversial goals/missed goals during high profile competitive matches. This has been a step in the right direction, however, there are still many issues in the world of soccer. 
The most contentious aspect of the sport is the offside rule and its many controversial calls or lack of calls. There have been countless instances when a player is wrongfully ruled offside after scoring a game changing goal. There have also been many cases in which a player has scored a game winning goal and the player was not ruled to be offside when the play should have been stopped. Incorrect offside calls have the potential to ruin games. Sometimes the linesmen, the referees in charge of judging offside calls, cannot see the passage of playing fast enough to make a correct decision. There are many cases in which a ball is played too quickly for anyone to make the correct call. Being similar to goal line technology, people have called out for offside technology to help the linesmen and to reduce the number of incorrect offside calls in a game. That is why the objective of this project is to create a working offside detection system that can accurately detect offside passes. Positional data will be exported from the system and a MATLAB script will be written so that it can analyze the positions of the players and the ball and determine if a pass was offside.

\section{Methods}

\section{1) The Offside Rule}

In order to understand how this offside detection system is going to function, one must understand the offside rule first. The offside rule is defined as follows [2]: "A player is offside when he/she is nearer to the opponent's goal line than the second to last opponent when the ball is played/passed from one player to the player that is nearest to the goal line. Being level with the second to last opponent does not constitute being offside, neither does being level with both the last two opponents if they happen to be in line". When judging the condition of being "nearer", only a player's head, torso, legs, and feet are taken into consideration. The player's arms do not count. A player cannot be offside in his/her own half of the pitch regardless of where he/she is positioned in relation to the ball or members of the opposing team. In order for a player to be considered offside, he/she must be involved in active play. This can be accomplished in two ways. One is to be interfering with the play, or if the player is interfering with an opponent.

\section{2) VICON System Specifications \& Setup}

A VICON motion tracking camera system [3] and software was used for the development of this system. The College of Arts \& Media currently has a VICON system in the Digital Animation Center (DAC). This VICON system consists of 20 Bonita 10 infrared tracking cameras and they are placed along the edges of the room. The cameras all face the center of the room where an $18^{\prime} \times 14^{\prime}$ space is designated for digital motion tracking. Each Bonita 10 camera has a maximum frame rate of $250 \mathrm{fps}$. The lens has an operating range of 13 meters and a maximum field of vision that is $70.29^{\circ} \times 70.29^{\circ}$. The DAC normally uses a VICON software called BLADE for their animation and motion capture needs. Fortunately, VICON also provides a software called Tracker which tracks the 
positions of rigid bodies and exports that data as a file, or in real time if needed. VICON was generous and provided a free month-long trial of their Tracker software for this project. The Bonita 10 cameras track rigid bodies via the use of reflective markers. In order to create a rigid body in the system, the reflective markers need to be grouped into unique clusters [4]. Each pattern must be unique, otherwise the cameras will confuse one cluster for another and combine or remove desired rigid bodies. Figure 1 shows some of the reflective markers used in this project.

Once the reflective markers have been put into unique clusters, the next step is to strap them onto the desired rigid bodies. In this project, the objects that need to be tracked are each player's legs, torso, and head. There are four players involved in this simulation, so a total of four heads, four torsos, and eight legs need to be tracked. Figure 2 shows an example of how the markers were strapped onto the players.

Once each player has been strapped with their designated markers, the next step is to use the Tracker software to create the rigid bodies. Once the cameras have been calibrated and set, the software will recognize each marker and each unique pattern and create a rigid body. Each rigid body can be labeled so that one can keep track of which rigid body belongs to each player. Figure 3 shows how each rigid body is represented in the software for each player and the ball.

Additionally, the ball that will be used during this project also needs to be tracked by the VICON camera system. Since the reflective markers that are used to track the players are spheres, they cannot be used to track the ball. The reflective markers would keep the ball from rolling. The solution to this problem was

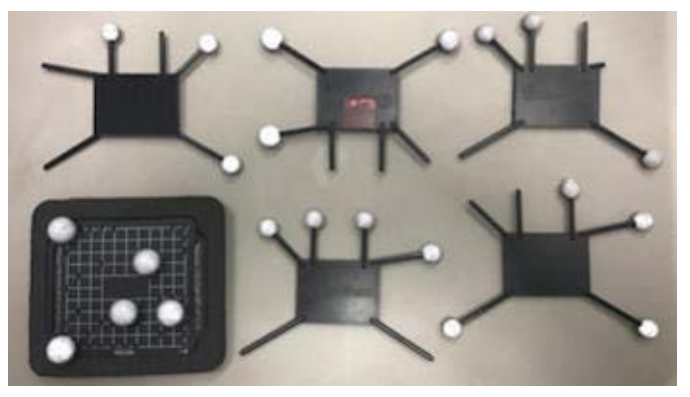

Figure 1. Reflective markers for rigid body tracking.
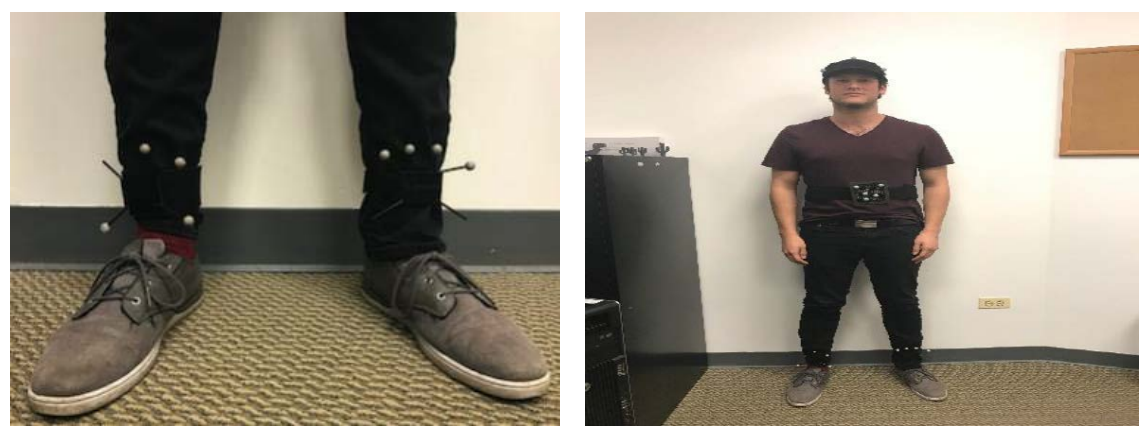

Figure 2. Reflective marker set up. 


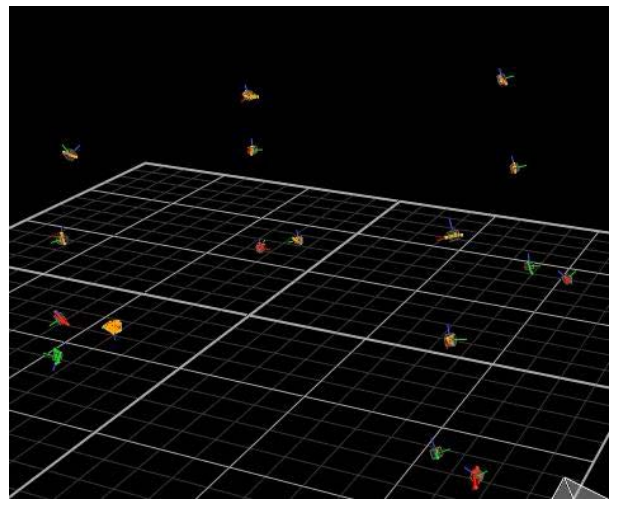

Figure 3. Rigid bodies as shown on VICON Tracker software.

to use OptiTrack reflective tape. The reflective tape was cut into small circles and placed in unique patterns across the ball so that the software could identify the ball as its own rigid body. Figure 4 shows the ball and the reflective tape used for tracking.

\section{3) Single Pass Simulation}

In this project, simulations were conducted with four players. Players 1 and 2 were part of Team A. Team A was the attacking team making the passes. Players 3 and 4 were part of Team B. Team B was the defending team setting the offside line. Figure 5 shows the position of both teams before and after the single pass was made.

It can be seen that the only object that moves is the ball. This was a way to control the offside line in order to ensure that they algorithm correctly identified the type of pass. In this situation, the ball is at rest and is passed only once. This simulation was conducted twice. Once for an onside pass and once for an offside pass. In the first pass, player 1 passes the ball to player 2. Both players are behind players 3 and 4, and therefore onside. In the second pass, player 2 is in front of players 3 and 4 and therefore in an offside position. Figure 6 shows the loop in the algorithm that was used to determine the frames in which the ball was moving and shows the first 22 frames.

The loop runs through all the position changes in the X-direction of the ball and finds any frame where the ball has traveled more than 10 millimeters. Once it has found these frames, it places them in the vector shown in Figure 6. The first frame in the vector is where the pass was made. The rest of the algorithm would then find the player's position at this frame and determine if the pass was onside or offside. This report will not go into further detail about the methods used for the single pass scenarios. This report will instead have an in depth look at the methods used for the final running simulation [5].

\section{4) Final Running Simulation}

Once the code had been created for a single pass, the next step was to modify the algorithm and run a simulation where all players are constantly changing positions, thus changing the offside line. Figure 7 shows an example of the changes in player position for the final simulation. 


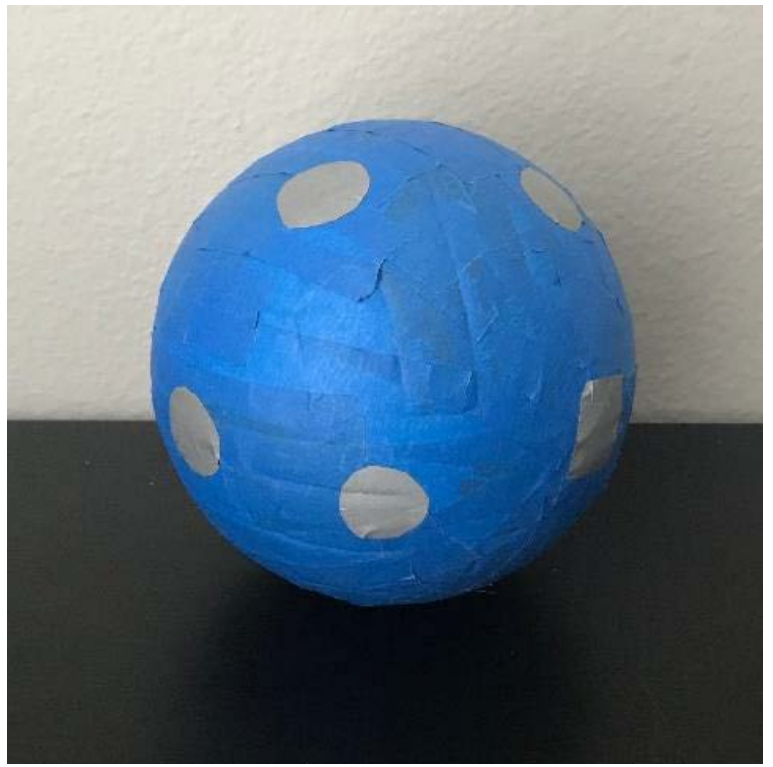

Figure 4. Ball and its reflective markers.
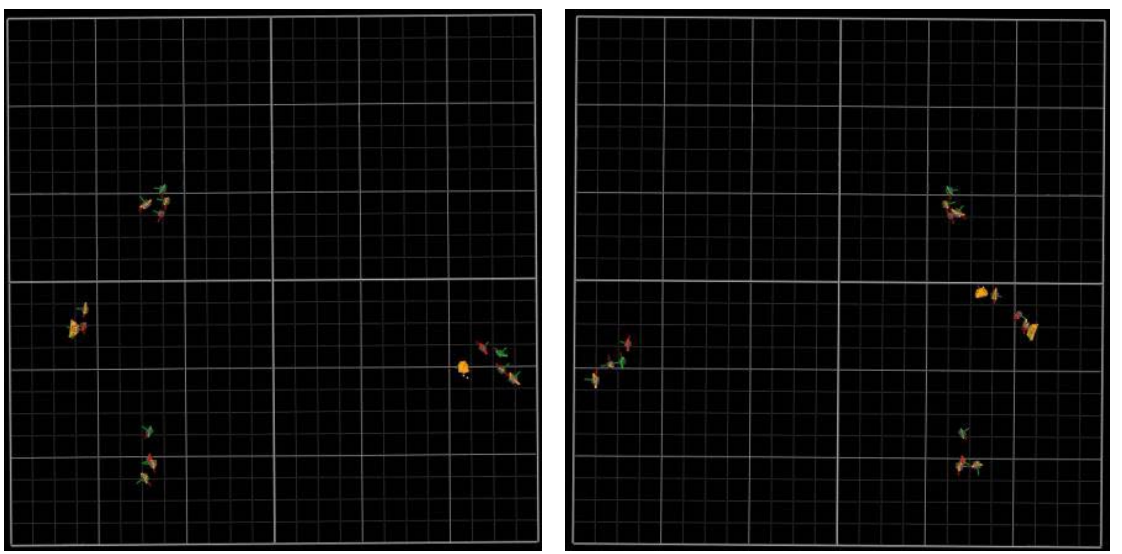

Figure 5. Static trial player positions before \& after pass.

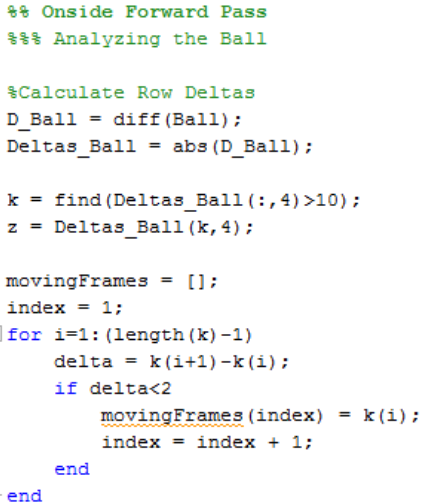

fo Onside Forward Pass

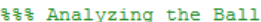

SCalculate Row Deltas

D_Ball = $\operatorname{diff}($ Ball);

Deltas_Ball = abs (D_Ball);

$k=$ find $($ Deltas_Ball $(:, 4)>10)$;

$z=\operatorname{Deltas} \operatorname{Ball}(k, 4)$

movingFrames $=[]$;

index $=1$;

for $i=1$ : (length $(k)-1$ )

delta $=k(i+1)-k(i)$.

if delta<2

movingFrames (index) $=\mathrm{k}(\mathrm{i})$;

index $=$ index +1 ;

end

end

movingFrames = movingFrames';

s. Pass is started at movingFrames(1) at this point in the code

sonow need to compare the positions of all players at the index/frame..

stwhen pass was made, i.e if pass made at frame 466 . Find position..

sof all players at frame 466 and compare.

\begin{tabular}{|c|c|c|}
\hline & movingFrames & $x$ \\
\hline & $123 \times 1$ double & \\
\hline & 1 & 2 \\
\hline 1 & 157 & \\
\hline 2 & 158 & \\
\hline 3 & 159 & \\
\hline 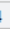 & 160 & \\
\hline 5 & 161 & \\
\hline 5 & 162 & \\
\hline 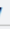 & 163 & \\
\hline 3 & 164 & \\
\hline 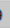 & 165 & \\
\hline 0 & 166 & \\
\hline 1 & 167 & \\
\hline 2 & 168 & \\
\hline 3 & 169 & \\
\hline 4 & 170 & \\
\hline 5 & 171 & \\
\hline 6 & 172 & \\
\hline 7 & 173 & \\
\hline 8 & 174 & \\
\hline 9 & 175 & \\
\hline 0 & 176 & \\
\hline 1 & 177 & \\
\hline 2 & 178 & \\
\hline
\end{tabular}

Figure 6. Loop to determine moving frames. 

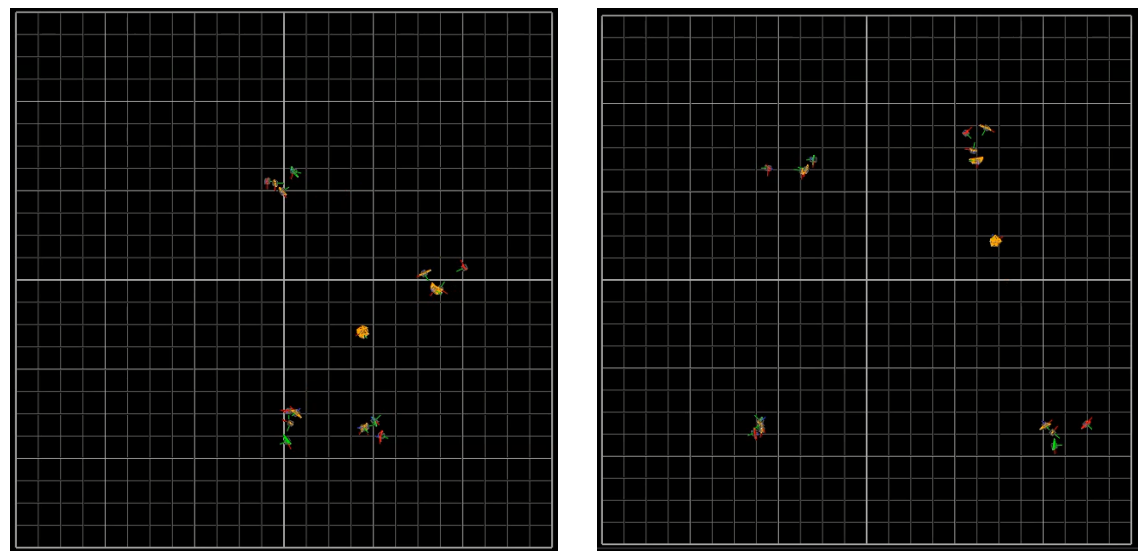

Figure 7. Running simulation player positions changing with time.

This running simulation is the final run that is to be analyzed by the algorithm. For this project, the running simulation lasted 2 minutes and 9 seconds. The code was altered so that it could read all passes sequentially. In order for the algorithm to detect passes, the positional data needed to be loaded onto MATLAB. The data for each rigid body was exported as an excel file. Figure 8 shows the way the data was formatted for all rigid bodies.

The software tracks six different types of positional data for each rigid body. Columns one through three are the rotational positions in the $\mathrm{X}, \mathrm{Y}$, and $\mathrm{Z}$ directions. These are measured in radians. Columns four through six are the translational positions in the $\mathrm{X}, \mathrm{Y}$, and $\mathrm{Z}$ directions. These are measured in millimeters. This project will only focus on columns four through six for each rigid body since the translational data is what will be analyzed. In this simulation, the ball was passed under three different conditions. The first type of pass that was observed was when the ball was at rest and was then kicked. In order to determine when the ball was kicked, the code looks at the changes in the X-direction. If the changes in the X-direction went from less than $10 \mathrm{~mm}$ per frame to greater than $10 \mathrm{~mm}$ per frame, then the ball was considered to be in motion, and thus passed at that specific frame. Figure 9 shows the changes in the X-direction for the ball at frame 2944.

The majority of the passes happened under these conditions. Figure 10 shows how the code was written to capture the frames where these pass conditions were met.

The second type of pass that was observed was when the ball was passed vertically. In this scenario, the code needed to read the changes in the Y-direction for the ball. The same conditions were applied as in the first type of pass. If the changes in the Y-direction went from less than $10 \mathrm{~mm}$ per frame to greater than $10 \mathrm{~mm}$ per frame, then the ball was considered to be in motion, thus passed at that specific frame. Figure 11 shows the changes in the $\mathrm{Y}$-direction for the ball.

Only one pass fell under this criterion and that was at frame 561. Figure 12 shows how the code was written to determine the frame where this pass condition was met. 


\begin{tabular}{|r|r|r|r|r|r|r|r|r|}
\hline & & \multicolumn{9}{|c|}{ Global Angle Player_3_Head: Player_3_Head } & & \\
\hline Frame & Sub Frame & RX & RY & RZ & TX & \multicolumn{1}{l|}{ TY } & \multicolumn{1}{l|}{ TZ } \\
\hline & & rad & rad & rad & mm & mm & \multicolumn{1}{l|}{ mm } \\
\hline 1 & 0 & -0.420662 & 0.539482 & 4.0564 & 697.985 & -1718.96 & 1613.23 \\
\hline 2 & 0 & -0.397319 & 0.521966 & 4.04231 & 701.481 & -1716.14 & 1613.43 \\
\hline 3 & 0 & -0.407936 & 0.531558 & 4.04906 & 705.444 & -1714.86 & 1613.23 \\
\hline 4 & 0 & -0.39644 & 0.514076 & 4.043 & 709.124 & -1711.97 & 1613.28 \\
\hline 5 & 0 & -0.406556 & 0.516764 & 4.04654 & 712.853 & -1710.84 & 1612.92 \\
\hline
\end{tabular}

Figure 8. Exported positional data for player 3 head first five frames.

\begin{tabular}{|r|r|r|r|r|r|r|}
\hline & \multicolumn{1}{|c|}{1} & \multicolumn{1}{|c|}{2} & \multicolumn{1}{|c|}{3} & \multicolumn{1}{l|}{4} & \multicolumn{1}{l|}{5} & \multicolumn{1}{l|}{6} \\
\hline 2932 & 0.0012 & 0.0018 & 0.0025 & 0.0610 & 0.0320 & 0.2031 \\
\hline 2933 & $5.5000 \mathrm{e}-04$ & 0.0024 & $5.8300 \mathrm{e}-04$ & 0.1090 & 0.1640 & 0.0281 \\
\hline 2934 & 0.0044 & $1.0000 \mathrm{e}-05$ & 0.0019 & 0.0280 & 0.2130 & 0.1217 \\
\hline 2935 & 0.0024 & $9.6000 \mathrm{e}-04$ & 0.0045 & 0.1870 & 0.2850 & 0.2043 \\
\hline 2936 & 0.0011 & $4.0000 \mathrm{e}-05$ & $7.7900 \mathrm{e}-04$ & 0.0070 & 0.0010 & 0.0414 \\
\hline 2937 & 0.0011 & 0.0018 & 0.0016 & 0.0960 & 0.2620 & 0.0774 \\
\hline 2938 & 0.0012 & 0.0030 & 0.0036 & 0.0860 & 0.2530 & 0.0159 \\
\hline 2939 & 0.0021 & 0.0055 & 0.0028 & 0.0060 & 0.3590 & 0.3641 \\
\hline 2940 & 0.0018 & 0.0020 & 0.0088 & 0.1220 & 0.6420 & 0.0041 \\
\hline 2941 & 0.0016 & 0.0023 & 0.0024 & 0.1680 & 0.3130 & 0.0936 \\
\hline 2942 & $2.5000 \mathrm{e}-04$ & 0.0055 & $2.1100 \mathrm{e}-04$ & 0.0920 & 0.3540 & 0.2038 \\
\hline 2943 & $1.0000 \mathrm{e}-04$ & 0.0099 & 0.0145 & 0.2730 & 0.9080 & 0.0635 \\
\hline 2944 & 0.0318 & 0.0365 & 0.0288 & 11.6370 & 6.0090 & 3.6835 \\
\hline 2945 & 0.0730 & 0.1185 & 0.0129 & 24.9890 & 8.1310 & 1.8572 \\
\hline 2946 & 0.0723 & 0.0985 & 0.0070 & 24.2270 & 9.2310 & 1.8648 \\
\hline 2947 & 0.0725 & 0.1002 & 0.0233 & 24.8710 & 9.9680 & 0.1990 \\
\hline 2948 & 0.0852 & 0.0507 & 0.0213 & 24.5110 & 10.1580 & 1.0405 \\
\hline 2949 & 0.0759 & 0.0673 & 0.0022 & 24.7050 & 11.0640 & 0.6678 \\
\hline 2950 & 0.0691 & 0.0654 & 0.0026 & 25.3650 & 11.5280 & 1.0138 \\
\hline 2951 & 0.0937 & 0.0511 & 0.0293 & 24.6610 & 9.6710 & 1.8980 \\
\hline 2952 & 0.0784 & 0.0429 & 0.0103 & 24.7840 & 11.1140 & 2.6512 \\
\hline 2953 & 0.0836 & 0.0431 & 0.0161 & 24.6250 & 10.7820 & 3.3800 \\
\hline & & & & & & \\
\hline
\end{tabular}

Figure 9. Column 4 shows ball changes in X-direction at frame 2944 for pass Type 1.

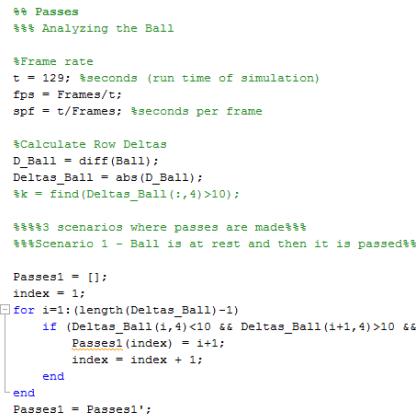

Figure 10. Pass type 1 code for determining relevant frames.

\begin{tabular}{|r|r|r|r|r|r|r|}
\hline & \multicolumn{1}{|c|}{1} & \multicolumn{1}{|c|}{2} & \multicolumn{1}{|c|}{3} & \multicolumn{1}{l|}{4} & \multicolumn{1}{l|}{5} & \multicolumn{1}{l|}{6} \\
\hline 550 & 0.0042 & 0.0091 & 0.0024 & 1.0030 & 0.0200 & 0.0260 \\
\hline 551 & 0.0174 & $4.9100 \mathrm{e}-04$ & $3.4000 \mathrm{e}-04$ & 0.4040 & 0.3200 & 0.0870 \\
\hline 552 & $2.2000 \mathrm{e}-04$ & $7.7800 \mathrm{e}-04$ & 0.0080 & 0.9820 & 0.4000 & 0.2222 \\
\hline 553 & 0.0060 & 0.0032 & $3.3000 \mathrm{e}-04$ & 1 & 0.3000 & 0.1190 \\
\hline 554 & 0.0125 & 0.0115 & 0.0161 & 1.7350 & 0.2700 & 0.1800 \\
\hline 555 & 0.0024 & 0.0027 & 0.0056 & 0.7820 & 0.9800 & 0.0740 \\
\hline 556 & 0.0061 & 0.0082 & 0.0016 & 1.2710 & 0.2200 & 0.0650 \\
\hline 557 & 0.0103 & 0.0014 & 0.0073 & 0.2160 & 0.4200 & 0.0390 \\
\hline 558 & 0.0242 & 0.0068 & $2.8000 \mathrm{e}-04$ & 0.0230 & 0.0500 & 0.5350 \\
\hline 559 & 0.0048 & 0.0023 & 0.0012 & 0.9080 & 0.4500 & 0.0250 \\
\hline 560 & 0.0062 & 0.0026 & 0.0018 & 0.5910 & 0.7900 & 0.2580 \\
\hline 561 & 0.0541 & 0.0466 & 0.0026 & 2.1030 & 13.9800 & 0.5970 \\
\hline 562 & 0.0285 & 0.0544 & 0.0041 & 5.4150 & 28.8100 & 0.0150 \\
\hline 563 & 0.0423 & 0.0862 & 0.0192 & 5.2470 & 28.4900 & 1.4840 \\
\hline 564 & 0.0514 & 0.0852 & 0.0128 & 4.9480 & 28.4100 & 0.2110 \\
\hline 565 & 0.0389 & 0.0859 & 0.0124 & 5.0970 & 28.4600 & 0.4200 \\
\hline 566 & 0.0284 & 0.0841 & 0.0092 & 5.4350 & 28.9700 & 1.3770 \\
\hline 567 & 0.0561 & 0.1063 & 0.0094 & 5.0590 & 27.9300 & 1.9270 \\
\hline 568 & 0.0776 & 0.1027 & 0.0101 & 3.4800 & 28.6500 & 0.1220 \\
\hline 569 & 0.0279 & 0.1110 & 0.0296 & 5.2140 & 28.7300 & 1.3040 \\
\hline & & & & & & \\
\hline
\end{tabular}

Figure 11. Column 5 shows ball changes in Y-direction at frame 561 for pass type 2. 


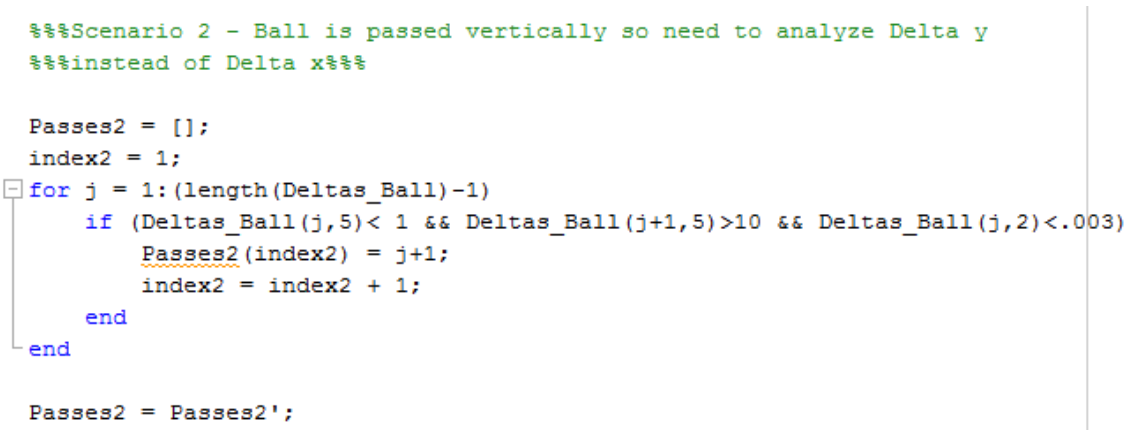

Figure 12. Pass type 2 code for determining relevant frame.

The final type of pass that was observed was when the ball had already been passed and was passed again by the receiving player while the ball was still in motion. In this scenario, the ball was never at rest between passes and thus needed to be analyzed in a different way. For this scenario, the velocities of the ball at each frame were determined. If the changes in the X-direction were greater than $10 \mathrm{~mm}$ per frame, but there was a sign change in the velocities, then the frames where the sign changes occurred were considered a pass. Figure 13 shows the changes in the X-direction for the ball at frame 2304 .

There is a sign change in the velocities at frame 2304 (Figure 14). This means that a pass was made at this frame. Figure 15 shows how the code was written to calculate the velocities at each frame and how the sign changes were found.

Finally, once pass frames had been isolated, various loops were created in the algorithm that would compare the positions of each player's legs, torso, and head at those frames. A final loop was created to compare those positions between the attacking players and the defending players. Figure 16 shows how the player's positions at all relevant frames were found.

The final section in the algorithm then determined if the pass was onside or offside. Figure 17 shows the final loops in the code that determined offside passes.

\section{Results}

The results for the single pass simulations can be seen on Table 1.

The results determined from the algorithm match the results that were obtained by visually confirming the pass from the simulation videos that were recorded from the software.

The running simulation is more complex than the single pass simulations. In order to determine the number of passes and their status, the frames where the passes were made needed to be determined. Table 2 shows the number of passes and their corresponding frames.

There were 45 passes in total throughout this simulation. However due to glitches in the system as well as gaps in the data due to camera limitations, only 43 out of the 45 passes were recognized by the cameras. This is further explained in the Discussion section of this report. The missing passes did not affect the 


\begin{tabular}{|c|c|c|c|c|c|c|}
\hline & 1 & 2 & 3 & 4 & 5 & 6 \\
\hline 2296 & 0.0202 & 0.0679 & 0.0040 & 12.0100 & 2.9770 & 7.1770 \\
\hline 2297 & 0.0229 & 0.2066 & 0.0189 & 13.8700 & 4.2330 & 11.6390 \\
\hline 2298 & 0.0351 & 0.2045 & 0.0156 & 15.9900 & 3.1270 & 11.4400 \\
\hline 2299 & 0.0193 & 0.0993 & 0.0122 & 15.9300 & 4.7620 & 7.0960 \\
\hline 2300 & 0.0439 & 0.0904 & 0.0094 & 16.1900 & 3.9970 & 6.4670 \\
\hline 2301 & 0.6299 & 0.0700 & 0.7086 & 55.4400 & 17.8960 & 32.0290 \\
\hline 2302 & 0.0792 & 0.1349 & 0.0197 & 15.0100 & 1.5640 & 0.7700 \\
\hline 2303 & 0.0715 & 0.1571 & 0.0723 & 12.4900 & 3.1340 & 0.2990 \\
\hline 2304 & 0.0167 & 0.0334 & 0.0070 & 19.1200 & 1.8270 & 2.2880 \\
\hline 2305 & 0.0718 & 0.1268 & 0.0452 & 36.1600 & 6.0230 & 2.5560 \\
\hline 2306 & 0.0323 & 0.1060 & 0.1390 & 33.1600 & 2.4410 & 2.0390 \\
\hline 2307 & 0.0415 & 0.1446 & 0.1451 & 31.5400 & 2.8470 & 0.5460 \\
\hline 2308 & 0.0592 & 0.1138 & 0.1212 & 32.4200 & 0.6880 & 0.8540 \\
\hline 2309 & 0.0691 & 0.1274 & 0.1290 & 31.6600 & 1.2480 & 1.7510 \\
\hline 2310 & 0.1152 & 0.1336 & 0.1642 & 29.8500 & 2.6560 & 0.9710 \\
\hline 2311 & 0.0888 & 0.1467 & 0.1136 & 31.2200 & 0.3010 & 3.5010 \\
\hline 2312 & 0.0581 & 0.1643 & 0.0935 & 31.0600 & 0.6410 & 4.5100 \\
\hline 2313 & 0.1358 & 0.1736 & 0.1073 & 29.2000 & 0.3300 & 1.0740 \\
\hline
\end{tabular}

Figure 13. Columns 4 shows ball changes in X-direction at frame 2304 for pass type 3.

\begin{tabular}{|l|c|}
\hline & 1 \\
\hline 2296 & $-1.2072 e+03$ \\
\hline 2297 & $-1.3942 e+03$ \\
\hline 2298 & $-1.6073 e+03$ \\
\hline 2299 & $-1.6013 e+03$ \\
\hline 2300 & $-1.6274 e+03$ \\
\hline 2301 & $-5.5728 e+03$ \\
\hline 2302 & $-1.5088 e+03$ \\
\hline 2303 & $-1.2555 e+03$ \\
\hline 2304 & $1.9219 e+03$ \\
\hline 2305 & $3.6348 e+03$ \\
\hline 2306 & $3.3332 e+03$ \\
\hline 2307 & $3.1704 e+03$ \\
\hline 2308 & $3.2588 e+03$ \\
\hline 2309 & $3.1824 e+03$ \\
\hline 2310 & $3.0005 e+03$ \\
\hline 2311 & $3.1382 e+03$ \\
\hline 2312 & $3.1221 e+03$ \\
\hline 2313 & $2.9352 e+03$ \\
\hline
\end{tabular}

Figure 14. Shows the calculated velocities for the frames around frame 2304.

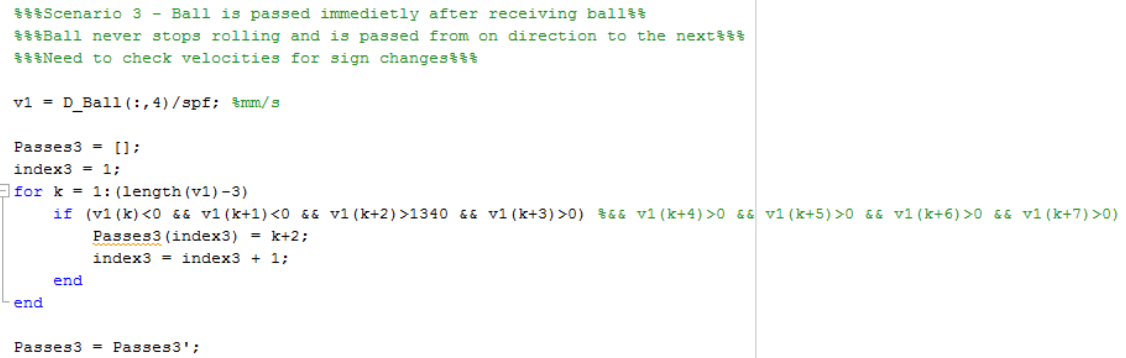

Figure 15. Pass type 3 code for determining relevant frames.

Table 1. Results for the single pass simulations.

\begin{tabular}{cccc}
\hline \multicolumn{2}{c}{ Onside Static Pass } & \multicolumn{2}{c}{ Offside Static Pass } \\
\hline Frame & Status & Frame & Status \\
\hline \multirow{2}{*}{466} & Onside & 157 & Offside \\
\hline
\end{tabular}




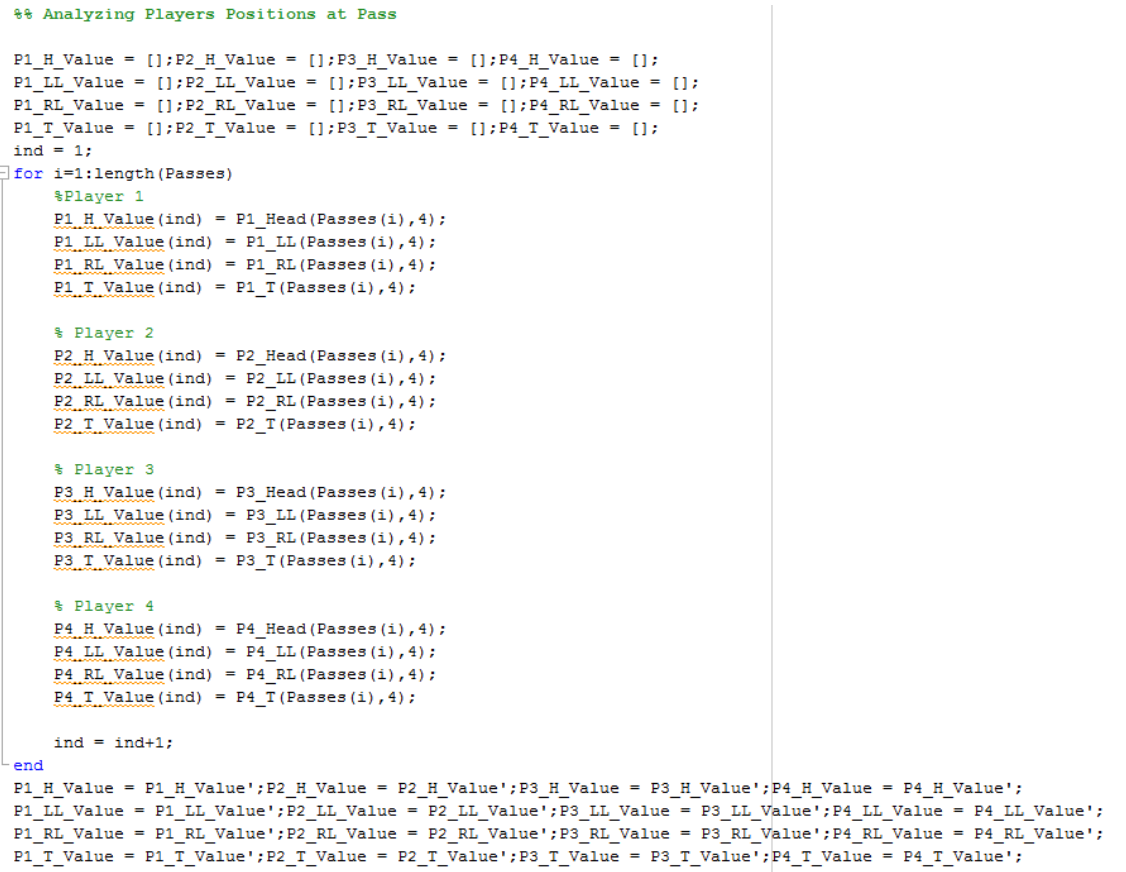

Figure 16. Code for determining player positions at relevant frames.

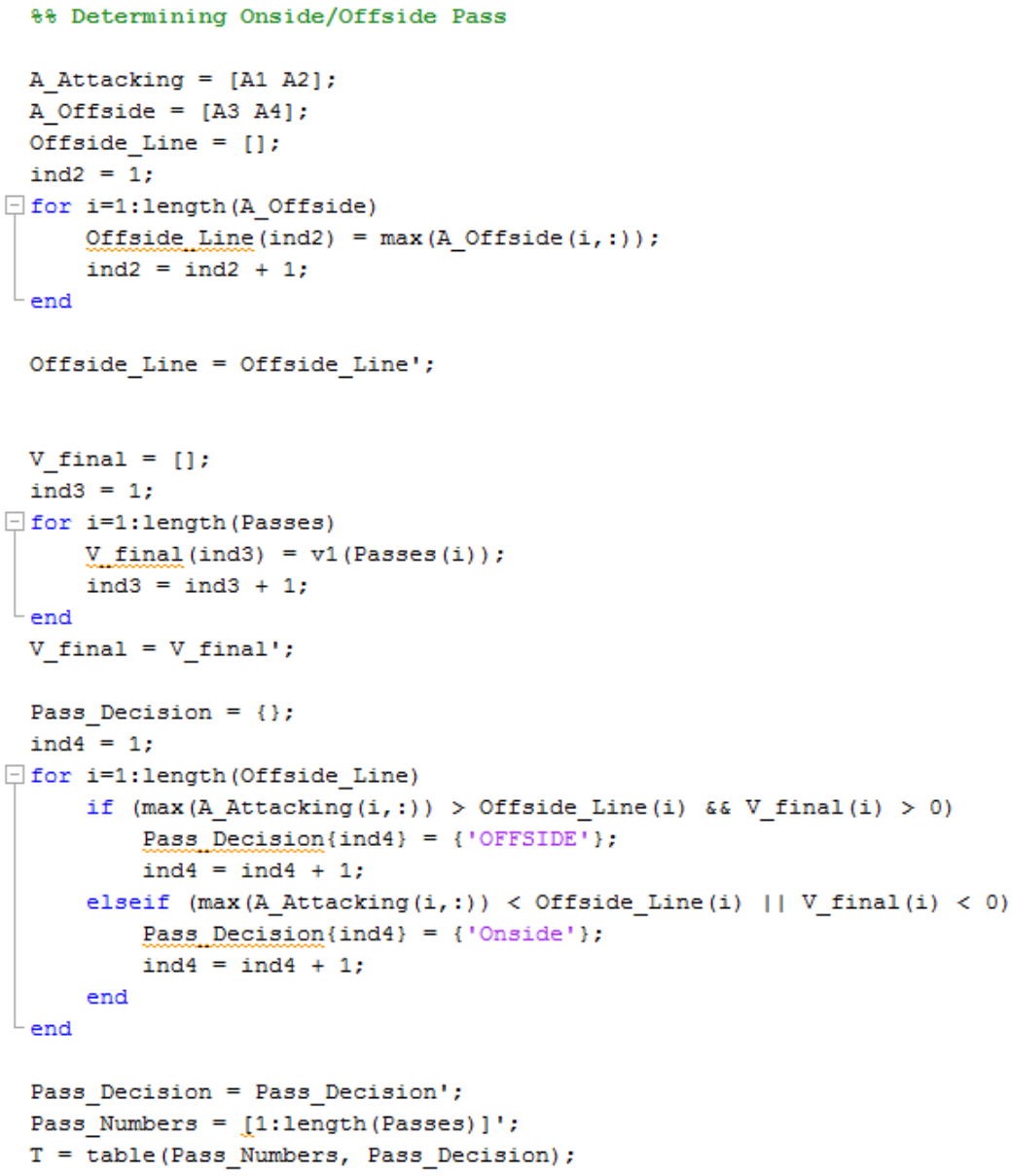

Figure 17. Final loops for determining offside passes. 
Table 2. Number of passes \& corresponding frames their corresponding frames.

\begin{tabular}{cccccccccc}
\hline Pass & Status & Pass & Status & Pass & Status & Pass & Status & Pass & Status \\
\hline 1 & 108 & 11 & 2944 & 21 & 5687 & 31 & 8771 & 41 & 12,037 \\
2 & 329 & 12 & 3206 & 22 & 5957 & 32 & 9591 & 42 & 12,320 \\
3 & 561 & 13 & 3446 & 23 & 6178 & 33 & 10,039 & 43 & 12,420 \\
4 & 903 & 14 & 3723 & 24 & 6398 & 34 & 10,347 \\
5 & 1178 & 15 & 4016 & 25 & 6612 & 35 & 10,574 \\
6 & 1354 & 16 & 4342 & 26 & 7105 & 36 & 10,854 \\
7 & 1631 & 17 & 4621 & 27 & 7569 & 37 & 11,120 \\
8 & 1869 & 18 & 4907 & 28 & 7836 & 38 & 11,362 \\
9 & 2144 & 19 & 5218 & 29 & 8237 & 39 & 11,612 \\
10 & 2304 & 20 & 5485 & 30 & 8468 & 40 & 11,781 \\
\hline
\end{tabular}

results of this project. Once the number of passes and their corresponding frames had been identified, the algorithm was able to determine whether each pass was onside or offside. Table 3 shows the final results of the running simulation for each pass. Table 4 shows the results from the visual confirmation of each pass and is favorably compared to the simulation results given in Table 4.

\section{Conclusion}

In conclusion, the offside detection system prototype has successfully identified onside and offside passes. Table 3 and Table 4 in the results section are identical. This means that the algorithm accurately determined the status of all 43 passes in the simulation. The success of the prototype is a major step towards the advancement of technology in soccer.

\section{Discussion}

Even though the prototype was a success, there were some limitations to the design. The biggest limitation to the design was the fact that the Tracker software was not meant to track a large number of rigid bodies at once. Since each rigid body had to be comprised of a unique pattern of reflective markers, it became difficult to have enough different patterns for each rigid body as the number of rigid bodies increased. Additionally, the software would sometimes confuse one rigid body for another if two players got to close to one another. If one player ran past another, there were instances where the markers on one player's leg were too close to another player's leg, thus creating one rigid body that the software could not recognize. As a result, there are gaps in the data that was exported from the software. Fortunately, these gaps did not occur at the instances where the passes were made. However, moving forward, this could be an issue if the gaps were to occur during important sections of the simulations.

Gaps in the data did not only occur when multiple rigid bodies got too close to one another. They also occurred whenever the ball was in motion. Since the 
Table 3. Pass results from algorithm.

\begin{tabular}{|c|c|}
\hline $\begin{array}{c}1 \\
\text { Pass_Numbers }\end{array}$ & $\begin{array}{c}2 \\
\text { Pass_Decision }\end{array}$ \\
\hline 1 & Onside \\
\hline 2 & Onside \\
\hline 3 & Onside \\
\hline 4 & Onside \\
\hline 5 & Offside \\
\hline 6 & Onside \\
\hline 7 & Onside \\
\hline 8 & Onside \\
\hline 9 & Onside \\
\hline 10 & Offside \\
\hline 11 & Onside \\
\hline 12 & Onside \\
\hline 13 & Onside \\
\hline 14 & Offside \\
\hline 15 & Onside \\
\hline 16 & Onside \\
\hline 17 & Onside \\
\hline 18 & Offside \\
\hline 19 & Onside \\
\hline 20 & Offside \\
\hline 21 & Onside \\
\hline 22 & Onside \\
\hline 23 & Offside \\
\hline 24 & Onside \\
\hline 25 & Offside \\
\hline 26 & Onside \\
\hline 27 & Onside \\
\hline 28 & Onside \\
\hline 29 & Offside \\
\hline 30 & Onside \\
\hline 31 & Offside \\
\hline 32 & Onside \\
\hline 33 & Offside \\
\hline 34 & Onside \\
\hline 35 & Offside \\
\hline 36 & Onside \\
\hline
\end{tabular}




\begin{tabular}{ll}
\hline 37 & Offside \\
38 & Onside \\
39 & Offside \\
40 & Onside \\
41 & Onside \\
42 & Onside \\
43 & Onside \\
\hline
\end{tabular}

Table 4. Pass results from visual confirmation of simulation recordings.

\begin{tabular}{cccccccccc}
\hline Pass & Status & Pass & Status & Pass & Status & Pass & Status & Pass & Status \\
\hline 1 & Onside & 11 & Onside & 21 & Onside & 31 & OFFSIDE & 41 & Onside \\
2 & Onside & 12 & Onside & 22 & Onside & 32 & Onside & 42 & Onside \\
3 & Onside & 13 & Onside & 23 & OFFSIDE & 33 & OFFSIDE & 43 & Onside \\
4 & Onside & 14 & OFFSIDE & 24 & Onside & 34 & Onside \\
5 & OFFSIDE & 15 & Onside & 25 & OFFSIDE & 35 & OFFSIDE \\
6 & Onside & 16 & Onside & 26 & Onside & 36 & Onside \\
7 & Onside & 17 & Onside & 27 & Onside & 37 & OFFSIDE \\
8 & Onside & 18 & OFFSIDE & 28 & Onside & 38 & Onside \\
9 & Onside & 19 & Onside & 29 & OFFSIDE & 39 & OFFSIDE \\
10 & OFFSIDE & 20 & OFFSIDE & 30 & Onside & 40 & Onside \\
\hline
\end{tabular}

software needs to see all reflective tape marks on the ball in order to establish it as a rigid body, the bottom portion of the ball did not have any reflective tape on it. This resulted in gaps in the data whenever the ball rolled since the bottom portion of the ball would end up on top while in motion. When this happened, the cameras would momentarily lose track of the ball for a few frames. The cameras would detect the ball once it had completed a rotation and all pieces of tape were visible yet again. These gaps in the data affected two of the passes during the simulation. Frames 9175 and 12,202 saw two short and quick passes between players 1 and 2 . Since there were gaps in the data, the software did not track these passes. Fortunately, both of these passes were onside and happened at a short distance where they did not affect the results of the simulation. All offside passes were accurately tracked and were not affected by the gaps in the data. Additionally, there were some movement restrictions throughout this project. The VICON system was located inside a computer lab/classroom, therefore all passes needed to remain on the ground. The ball needed to be passed at a reasonable speed so that no equipment or students would get injured.

Moving forward there are many improvements that could be made to this experimental offside detection system. The main improvement that can be made is to improve the camera system. The VICON system works great and the infrared 
tracking is accurate. However, in a real game setting this system will be impossible to use. The infrared tracking will simply not work in an outdoor environment. In order for the cameras to detect the reflective markers, there needs to be zero sunlight in the room and all other reflective surfaces need to be covered up. Additionally, in a real game setting it is not practical to have spherical trackers on the player's bodies. One solution is to utilize reflective tape on the players so that they can move as they normally would. However, this would only be practical in an indoor setting. The best way to move forward is to adopt a camera system that uses artificial intelligence and machine learning to detect color and track objects based on color. There have been numerous research studies done within the last 10 years and the technology is there. In a real game setting these cameras can be used outdoors and would be able to track the colors of each team's jerseys. The VICON system does however work accurately and effectively in terms of the scope of this project. This project is the foundation for what is meant to be a radical change in the world of soccer. Offside technology is years away from being perfected, however this project has demonstrated that it can be done accurately and effectively given the proper resources. The purpose of this project has been completed and the main objective has been met. The VICON infrared camera system can accurately detect offside passes. This technology can be used to aid the referees and officials to fairly influence the outcome of soccer games.

\section{Acknowledgements}

1) The authors would like to thank the University of Colorado Denver Digital Animation Center.

2) The support of VICON was appreciated.

\section{Conflicts of Interest}

The authors declare no conflicts of interest regarding the publication of this paper.

\section{References}

[1] The Hidden Technologies at the 2018 FIFA World Cup, June 42018. https://football-technology.fifa.com/en/blog/hidden-technologies-at-the-2018-fwc

[2] Laws of the Game-FIFA, 2015/2016. https://img.fifa.com/image/upload/datdz0pms85gbnqy4j3k.pdf

[3] VICON Motion Capture System. https://www.vicon.com/motion-capture

[4] Cortes, N., Blount, E., Ringleb, S. and Onate, J.A. (2011) Soccer-Specific Video Simulation for Improving Movement Assessment. Sports Biomechanics, 10, 22-34. https://doi.org/10.1080/14763141.2010.547591

[5] Bodenheimer, B., Rose, C., Rosenthal, S. and Pella, J. (1997) The Process of Motion Capture: Dealing with the Data. In: Computer Animation and Simulation, Springer-Verlag, Berlin, 3-18. https://doi.org/10.1007/978-3-7091-6874-5_1 